\title{
043
}

\section{PREVALENZA E FATTORI DI RISCHIO ASSOCIATI ALLE INFEZIONI DA CHLAMYDIA TRACHOMATIS - DATI PRELIMINARI}

\author{
Latino M.A., Rosso C., De Intinis G., De Maria D., Intorcia P.
}

\section{S. S. Dip. di Batteriologia, Az. Osp. O.I.R.M. - Sant'Anna - Torino}

L'infezione da Chlamydia trachomatis rappresenta una delle infezioni a trasmissione sessuale (IST) più diffusa in tutti i paesi industrializzati tra le donne di età inferiore ai 25 anni.

\section{Obiettivi e metodi:}

Scopo dello studio è stato quello di valutare la prevalenza dell'infezione nella popolazione torinese di età compresa tra 18 e 24 anni e di individuare i gruppi a maggior rischio. Le pazienti sono state arruolate attraverso i ginecologi ed i consultori familiari. I test sono stati eseguiti col kit TMA Gen Probe Amplified Chlamydia trachomatis (BioMérieux) previo raggruppamento in pools di 5 campioni. I pools risultati positivi sono stati quindi riesaminati come singoli campioni. Risultati:

Dal $1^{\circ}$ Marzo 2004 al 30 Aprile 2005 sono state esaminate 750 donne con una prevalenza dell'infezione pari al $10.5 \%$, dato questo statisticamente significativo $(\mathrm{p}<0.001)$ visto che nella fascia di età superiore (25-35 anni) la prevalenza scendeva al 2\%. 124 donne (16.5\%) hanno avuto più di un partner negli ultimi 6 mesi in questo gruppo la prevalenza dell'infezione è stata del $24.2 \%$ vs il $7 \%(\mathrm{p}<0.001)$ del gruppo che ha dichiarato un solo partner. Il $22.5 \%$ (169/750) ha avuto almeno un partner occasionale con una prevalenza del $20.7 \%$ vs il $6.5 \%(p<0.001)$ nelle donne che hanno avuto solo partners stabili.

Nel gruppo in cui il partner manifestava sintomi riferibili ad una uretrite la prevalenza era del $22.2 \%$ vs il $7.3 \%(p<0.001)$ nelle donne il cui partner era asintomatico.

Considerando la provenienza delle pazienti da Paesi ad alta incidenza di IST come l'Est europeo e l'Asia, la prevalenza è stata rispettivamente del 17.7 e del $16.7 /$ vs 1 ' $8.6 \%$ $(p<0.001)$ nelle pazienti originarie dell'Europa occidentale.

\section{Conclusioni:}

I dati preliminari ci permettono finora di individuare alcuni ulteriori fattori di rischio per le infezioni da Chlamydia trachomatis oltre alla giovane età che già di per sé rappresenta un noto fattore di rischio. 